\title{
Accession ID
}

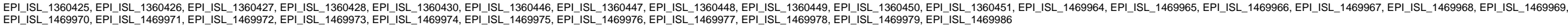
see above Child Health Research Foundation Child Health Research Foundatio EPI_ISL_1498127, EPI_ISL_1498131, EPI_ISL_1498132, EPI_ISL_1498136, EPI_ISL_1498137, EPI_ISL_1498138, EPI_ISL_1498139, EPI_ISL_1498140, EP_

See above
EPI_ISL_1508828, EPI_ISL_1508833, Institute for Developing Science and Health Initiatives (ideSHI)
EPI_ISL_1508084ional Institute of Laboratory Medicine and Referral Center

EPI ISL 1508891, EPI_ISL 1508892,
EPI ISL 1508893, EPI ISL 1508915

National Institute of Laboratory Medicine and Referral Center Genomic Research Lab, BCSIR

Genomic Research Lab. BCSIR

EPI_ISL_1508940, EPI_ISL_1508942, EPI'ISL_1508950, EPI_ISL-1508952, EPI_ISL_1508999

EPI_ISL_1509000, EPI_ISL_1509001,

EPI_ISL_1509298, EPI_ISL_1509299, EPIISL_1509637, EPI_ISL_1509924, EPI_ISL_1524771, EPI_ISL_1524775,
EPI_ISL_1524776, 2 PIIISL 1524802,
EPIIISL_1524872

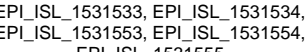
EPI_ISL_15_15566, EPI_ISL_1531557,

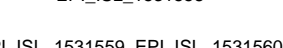

National Institute of Laboratory Medicine and Referral Center

National Institute of Laboratory Medicine and Referral Center

National Institute of Laboratory Medicine and Referral Center

National Institute of Laboratory Medicine and Referral Center

National Institute of Laboratory Medicine and Referral Center

National Institute of Laboratory Medicine and Referral Center

National Institute of Laboratory Medicine and Referral Center

National Institute of Laboratory Medicine and Referral Center

EPI_ISL_1531561, EPI_ISL_1531562

EPI_ISL_1531563, EPI_ISL_1531564

EPI_ISL_1531565, EPI_SL_1531566,
EPI_ISL_1531567

EPI_ISL_1531568, EPI_ISL_1531569

EPI ISL 1531570, EPIISL 1531571,
EPI ISL 1531572, EPI ISL-1531735

EPI_ISL_1531743, EPI_ISL_1531744, EPI_ISL_1532855, EPI_ISL_1532982,
EPI_ISL_1533423, EPIISL 1533426,
EPI_ISL_1533427 EPI_ISL_1533429, EPI_ISL_1533430,
EPI_ISL_1533728

EPI ISL_1533802, EPI ISL_1533852

EPI_ISL_1534610
National Institute of Laboratory Medicine and Referral Center

National Institute of Laboratory Medicine and Referral Center

National Institute of Laboratory Medicine and Referral Center

National Institute of Laboratory Medicine and Referral Center

National Institute of Laboratory Medicine and Referral Center

National Institute of Laboratory Medicine and Referral Center

National Institute of Laboratory Medicine and Referral Center

National Institute of Laboratory Medicine and Referral Center
Genomic Research Lab, BCSIR

Genomic Research Lab, BCSIR

Genomic Research Lab, BCSIR

Genomic Research Lab, BCSIR

Genomic Research Lab, BCSIR

Genomic Research Lab, BCSIR

Genomic Research Lab, BCSIR

Genomic Research Lab, BCSIR

Genomic Research Lab, BCSIR

Genomic Research Lab, BCSIR

Genomic Research Lab, BCSIR

Genomic Research Lab, BCSIR

Genomic Research Lab, BCSIR

Genomic Research Lab, BCSIR

Genomic Research Lab, BCSIR

Genomic Research Lab, BCSIR

Genomic Research Lab, BCSIR
CHRF Bangladesh Genomics Tean 498149, EPI_ISL_1498150, EPI_ISL_149815 Hassan Afrad, Sadia Rahman, Fidausi Qadri, Tahmina Shirin

Md. Murshed Hasan Sarkar, Shahina Akter, Abu Sayeed Mohammad Mahmud, Mohammad Samir Uzzaman, Eshrar Osman, Md. Ahasan Habib, Tanina
Akhter Banu, Barna Goswami, Iffat Jahan, Md. Saddam Hossain, Tasnim Nafisa, Md. Maruf Ahmed Molla, Mahmuda Yeasmin, Asish Kumar Ghosh, Arift Md. Murshed Hasan Sarkar, Shahina Akter, Abu Sayeed Mohammad Mahmud, Mohammad Samir Uzzaman, Eshrar Osman, Md. Ahasan Habib, Tanjina Akram, A. K. M. Shamsuzzaman, Md. Salim Khan Md. Murshed Hasan Sarkar, Shahina Akter, Abu Sayeed Mohammad Mahmud, Mohammad Samir Uzzaman, Eshrar Osman, Md. Ahasan Habib, Tanjina
Akhter Banu, Barna Goswami, Iffat Jahan, Md. Saddam Hossain, Tasnim Nafisa, Md. Maruf Ahmed Molla, Mahmuda Yeasmin, Asish Kumar Ghosh, Arifa Akram, A. K. M. Shamsuzzaman, Md. Salim Khan

Tasnim Nafisa, Md. Murshed Hasan Sarkar, Shahina Akter, Abu Sayeed Mohammad Mahmud, Mohammad Samir Uzzaman, Eshrar Osman, Md. Ahasan
Habib, Tanjina Akhter Banu, Barna Goswami, Iffat Jahan, Md. Saddam Hossain, Md. Maruf Ahmed Molla, Mahmuda Yeasmin, Asish Kumar Ghosh, Arifa Akram, A. K. M. Shamsuzzaman, Md Salim Khan Md. Marut Ahmed Molla, Md. Murshed Hasan Sarkar, Shahina Akter, Abu Sayeed Mohammad Mahmud, Mohammad Samir Uzzaman, Eshrar Osman, Mc
Ahasan Habib, Tanjina Akhter Banu, Barna Goswami, Ifrat Jahan, Md. Saddam Hossain, Tasnim Nafisa, Mahmuda Yeasmin, Asish Kumar Ghosh, Arifa
Akram, A. K. M. Shamsuzzaman, Md. Salim Khan Md. Murshed Hasan Sarkar, Shahina Akter, Abu Sayeed Mohammad Mahmud, Mohammad Samir Uzzaman, Eshrar Osman, Md. Ahasan Habib, Tanjina Akhter Banu, Barna Goswami, Iffat Jahan, Md. Saddam Hossain, Tasnim Nafisa, Md. Maruf Ahmed Molla, Mahmuda Yeasmin, Asish Kumar Ghosh, Ari Md. Murshed Hasan Sarkar, Shahina Akter, Abu Sayeed Mohammad Mahmud, Mohammad Samir Uzzaman, Eshrar Osman, Md. Ahasan Habib, Taniina
Akhter Banu, Barna Goswami, Iffat Jahan, Md. Saddam Hossain, Tasnim Nafisa, Md. Maruf Ahmed Molla, Mahmuda Yeasmin, Asish Kumar Ghosh, Arifta Akram, A. K. M. Shamsuzzaman, Md. Salim Khan
Md. Murshed Hasan Sarkar, Shahina Akter, Abu Sayeed Mohammad Mahmud, Mohammad Samir Uzzaman, Eshrar Osman, Md. Ahasan Habib, Tanjina Akhter Banu, Barna Goswami, Iffat Jahan, Md. Saddam Hossain, Tasnim Nafisa, Md. Marut Ahmed Molla, Mahmuda Yeasmin, Asish Kumar Ghosh, Aril Shahina Akter, Md. Murshed Hasan Sarkar, Abu Sayeed Mohammad Mahmud, Mohammad Samir Uzzaman, Eshrar Osman, Md. Ahasan Habib, Tanina
Akhter Banu, Barna Goswami, fffat Jahan, Md. Saddam Hossain, Tasnim Natisa, Md. Maruf Ahmed Molla, Mahmuda Yeasmin, Asish Kumar Ghosh, Arifa Akram, A. K. M. Shamsuzzaman, Md. Salim Khan
Tanjina Akhter Banu, Md. Murshed Hasan Sarkar, Abu Sayeed Mohammad Mahmud, Mohammad Samir Uzzaman, Eshrar Osman, Md. Ahasan Habib, Shahina Akter, Barna Goswami, Iffat Jahan, Md. Saddam Hossain, Mohammad Mohi Uddin, Tasnim Nafisa, Md. Ma
Asish Kumar Ghosh, Arifa Akram, A. K. M. Shamsuzzaman, Md. Salim Khan

Barna Goswami, Md. Murshed Hasan Sarkar, Abu Sayeed Mohammad Mahmud, Mohammad Samir Uzzaman, Eshrar Osman, Md. Ahasan Habib,
Shahina Akter, Tanijina Akher Yeasmin, Asish Kumar Ghosh, Arifa Akram, A. K. M. Shamsuzzaman, Md. Salim Khan

Iffat Jahan, Md. Murshed Hasan Sarkar, Abu Sayeed Mohammad Mahmud, Mohammad Samir Uzzaman, Eshrar Osman, Md. Ahasan Habib, Shahina Akter, Tanjina Akhter Banu, Barna Goswami, Md. Saddam Hossain, Mohammad Mohi Uddin, Tasnim Natisa, Md. M M
Asish Kumar Ghosh, Arifa Akram, A. K. M. Shamsuzzaman, Md. Salim Khan Md. Saddam Hossain, Md. Murshed Hasan Sarkar, Abu Sayeed Mohammad Mahmud, Mohammad Samir Uzzaman, Eshrar Osmann, Md. Ahasan Habib,
Shahina Akter, Tanjina Akhter Banu, Barna Goswami, Iffat Jahan, Mohammad Mohi dddin, Tassimi Nafisa, Md. Marut Ahmed Molla, Mahmuda Yeasmin, Md. Saddam Hossain, Md. Murshed Hasan Sarkar, Abu Sayeed Mohammad Mahmud, Mohammad Samir Uzzaman, Eshrar Osman, Md. Ahasan Habibb Mahmuda Yeasmin, Asish Kumar Ghosh, Arifa Akram, A. K. M. Shamsuzzaman, Md. Salim Khan
Mans Md. Murshed Hasan Sarkar, Abu Sayeed Mohammad Mahmud, Mohammad Samir Uzzaman, Eshrar Osman, Md. Ahasan Habib, Shahina Akter, Taninina
Akhter Banu, Barna Goswami, Iffat Jahan, Md. Saddam Hossain, Mohammad Mohi Udddin, Tasnim Nafisa, Md. Maruf Ahmed Molla, Mahmuda Yeasmin, Md. Murshed Hasan Sarkar, Shahina Akter, Abu Sayeed Mohammad Mahmud, Mohammad Samir Uzzaman, Eshrar Osman, Md. Ahasan Habib, Tanjina Asish Kumar Ghosh, Arifia Akram, A. K. M. Shamsuzzaman, Md. Salim Khan

Abu Sayeed Mohammad Mahmud, Md. Murshed Hasan Sarkar, Mohammad Samir Uzzaman, Eshrar Osman, Md. Ahasan Habib, Shahina Akter, Tanjina
Akhter Banu, Barna Goswami, Iffat Jahan, Md Saddam Hossain. Moham Asish Kumar Ghosh, Arifa Akram, A. K. M. Shamsuzzaman, Md. Salim Khan
A Sal

Mohammad Mohi Uddin, Md. Murshed Hasan Sarkar, Mohammad Samir Uzzaman, Eshrar Osman, Md. Ahasan Habib, Shahina Akter, Tanjina Akhter Banu, Abu Sayeed Mohammad Mahmud, Barna Gosswami, Iffat Jahan, Md. Saddam, Hossain, Mohammad Mohi Uddin, Tasnim Nafisa, Md. Maruf Ahmed
Molla, Mahmuda Yeasmin, Asish Kumar Ghosh, Arifa Akram, A. K. M. Shamsuzzaman, Md. Salim Khan Md. Murshed Hasan Sarkar, Shahina Akter, Abu Sayeed Mohammad Mahmud, Mohammad Samir Uzzaman, Eshrar Osman, Md. Ahasan Habib, Tanjina
Akhter Banu, Barna Goswami, Iffat Jahanan, Md. Saddam Hossain, Mohammad Mohi Uddain, Tasnim Nafisa, Md. Maruf Ahmed Molla, Mahmuda Yeasmin. 
EPI_ISL_1534611

EPI_ISL_1534612, EPI_ISL_1534613

EPI_ISL_1534614, EPI_ISL_1534615,
EPI ISL 1534616 EPI_ISL_1534617, EPI_ISL_1534618,

EPI_ISL_1534620, EPI_ISL_1534621

EPI_ISL_1538405

EPI ISL_1538406

EPIISL 1538407, EPIIISL_1538408,
EPI_ISL_1538409, EPIISL-1534410,

(1547065

EPI_ISL_1547372

EPI_ISL 1547828, EPI_ISL 1548044,
EPI_ISL_1548059, EPIISL_1548072

EPI_ISL_1575127 EPI_ISL 1582390, EPI_ISL_1582391,

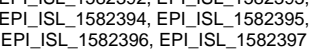
EPI_ISL 1583158, EPI_ISL1583188, EPI_ISL_1583252, EPI_ISL-1583253,
EPIISL1585269, EPIISL 158527,
EPI_ISL_1585940, EPI_ISL_1585942

EPIIISL_1587429, EPI_ISL_1587430, EP see above

EPI_ISL_1588437, EPI_ISL_1588593

EPI_ISL_1593804

EPI_ISL_1593853, EPI_ISL_1593854,
EPI ISL 1593855 EPI_ISL_1593856, EPI_ISL_1593857,

EPI_ISL_1593859, EPI_ISL1593860,
EPI_ISL_1593861, EPI_ISL_1593862 EPI ISL_1593863, EPI ISL_1593879

EPI_ISL_1593880, EPI_ISL_1593888 EPIISL_1593894, EPI_ISL_1595718,
EPIIISL_1595721, EPI ISL_1595831 EPI_ISL_1595832, EPI_ISL_1595833,
EPI_ISL_1595834 EPI ISL_1595835, EPI_ISL_1595837
National Institute of Laboratory Medicine and Referral Center

Institute for Developing Science and Health Initiatives (ideSHi) Institute for Developing Science and Health Initiatives (ideSHI) Institute of Epidemiology, Disease Control and Research Institute for Developing Science and Health Initiatives (ideSHi)

$$
\begin{gathered}
\text { Armed Forces Institute of Pathology (AFIP), Dhaka } \\
\text { Cantonment }
\end{gathered}
$$

Genomic Research Lab, BCSIR

Institute for Developing Science and Health Initiatives (ideSHi) Institute for Developing Science and Health Initiatives (ideSHi) Armed Forces Institute of Pathology (AFIP), Dhaka
Cantonment

Armed Forces Institute of Pathology (AFIP), Dhaka
Cantonment Genomic Research Lab, BCSIR

Genomic Research Lab, BCSIR

Institute for Developing Science and Health Initiatives (ideSHi) Institute for Developing Science and Health Initiatives (ideSHi) Armed Forces Institute of Pathology (AFIP), Dhaka
Cantonment

Genomic Research Lab, BCSIR Genomic Research Lab, BCSIR Armed Forces Institute of Pathology (AFIP), Dhaka
Cantonment Armed Forces Institute of Pathology (AFIP), Dhaka Genomic Research Lab, BCSIR Armed Forces Institute of Pathology (AFIP), Dhaka
Cantonment Genomic Research Lab, BCSIR Armed Forces Institute of Pathology (AFIP), Dhaka Armed Forces Institute of Pathology (AFIP), Dhaka
Cantonment Genomic Research Lab, BCSIR Genomic Research Lab, BCSIR Armed Forces Institute of Pathology (AFIP), Dhaka
Uzzaman, Eshrar Osman, Md. Ahasan Habib, Tanjina Akhter Banu, Barna Goswami, Iffat Jahan, Md. Saddam Hossain, Mohammad Mohi Uddin, Tasnim
Nafisa, Md. Marut Ahmed Molla, Mahmuda Yeasmin, Asish Kumar Ghosh, Arifa Akram, A. K. M. Shamsuzzaman, Md. Salim Khan Md. Murshed Hasan Sarkar, Shahina Akter, Abu Sayeed Mohammad Mahmud, Mohammad Samir Uzzaman, Eshrar Osman, Md. Ahasan Habib, Tanjina
Akhter Banu, Barna Goswami, Iffat Jahana, Md. Saddam Hossain, Mohammad Mohi Uddain, Tasnim Nafisa, Md. Maruf Ahmed Molla, Mahmuda Yeasmin, Md. Marut Ahmed Molla, Md. Murshed Hasan Sarkar, Shahina Akter, Abu Sayeed Mohammad Mahmud, Mohammad Samir Uzzaman, Eshrar Osman, Mc
Ahasan Habib, Tanjina Akhter Banu, Barna Gosswami, Iffat Jahan, Md. Saddam Hossain, Mohammad Mohi Uddin, Tasnim Nafisa, Mahmuda Yeasmin,
Asish Kumar Ghosh, Arifa Akram, A. K. M. Shamsuzzaman, Md. Salim Khan

Tasnim Nafisa, Md. Murshed Hasan Sarkar, Shahina Akter, Abu Sayeed Mohammad Mahmud, Mohammad Samir Uzzaman, Eshrar Osman, Md. Ahasan
Habib, Tanjina Akhter Banu, Barna Goswami, Iffat Jahan, Md. Saddam Hossain, Mohammad Mohi Uddin, Md. Maruf Ahmed Molla, Mahmuda Yeasmin

Shahina Akter, Md. Murshed Hasan Sarkar, Abu Sayeed Mohammad Mahmud, Mohammad Samir Uzzaman, Eshrar Osman, Md. Ahasan Habib, Tanina
Akhter Banu, Barna Goswami, Iffat Jahan, Md. Saddam Hossain, Mohammad Mohi Uddin, Tassim Nafisa, Md. Maruf Ahmed Molla, Mahmuda Yeasmin, Asish Kumar Ghosh, Arifa Akram, A. K. M. Shamsuzzaman, Md. Salim Khan Taninna Akhter Banu, Md. Murshed Hasan Sarkar, Abu Sayeed Mohammad Mahmud, Mohammad Samir Uzzaman, Eshrar Osman, Md. Ahasan Habib,
Shahina Akter, Barna Goswami, Iffat Jahan, Md. Saddam Hossain, Mohammad Mohi Uddin, Tasnim Nafisa, Md. Marut Ahmed Molla, Mahmuda Yeasmin, Tanijna Akhter Banu, Md. Murshed Hasan Sarkar, Abu Sayeed Mohammad Mahmud, Mohammad Samir Uzzaman, Eshrar Osman, Md. Ahasan Habib, Shanina Akter, Barna Goswami, Iffat Jahan, Md. Saddam Hossain, Mohammad Mohi Uddin, Tasnim Natisa, Md. Man
Asish Kumar Ghosh, Arifa Akram, A. K. M. Shamsuzzaman, Md. Salim Khan

Md. Murshed Hasan Sarkar, Abu Sayeed Mohammad Mahmud, Mohammad Samir Uzzaman, Eshrar Osman, Md. Ahasan Habib, Shahina Akter, Tanjina
Akhter Banu, Barna Goswami, ffiat Jahan, Md. Saddam Hossain, Mohammad Mohi Uddin, Tasnim Nafisa, Md. Maruf Ahmed Molla, Mahmuda Yeasmin,

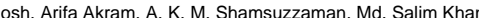

Iffat Jahan, Md. Murshed Hasan Sarkar, Abu Sayeed Mohammad Mahmud, Mohammad Samir Uzzaman, Eshrar Osman, Md. Ahasan Habib, Shahina

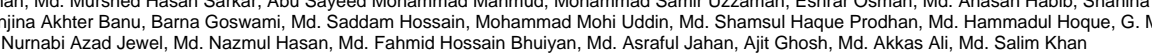
Mohammad Mohi Uddin, Md. Murshed Hasan Sarkar, Abu Sayeed Mohammad Mahmud, Mohammad Samir Uzzaman, Eshrar Osman, Md. Ahasan Habib,
Shahina Akter, Tanjina Akhter Banu, Barna Goswami, ffrat Jahan, Md. Saddam Hossain, Md. Kamrul slam, Md. Shamsul Haque Prodhan, Md. Hammadul Md. Murshed Hasan Sarkar, Abu Sayeed Mohammad Mahmud, Mohammad Samir Uzzaman, Eshrar Osman, Md. Ahasan Habib, Shahina Akter, Taninina
Akhter Banu, Barna Goswami, Iffat Jahan, Md. Saddam Hossain, Mohammad Mohi Uddin, Md. Kamrul Islam, Md. Shamsul Haque Prodhan, Md.
Hammadul Hoque, G. M. Nurnabi Azad Jewel, Md. Nazmul Hasan, Md. Fahmid Hossain Bhuiyan, Md. Asraful Jahan, Ajit Ghosh, Md. Akkas Ali, Md. Salim Hassan Afrad, Sadia Rahman, Fidausi Qadri, Tahmina Shirin Hassan Afrad, Sadia Rahman, Fidausi Qadri, Tahmina Shirin Md. Murshed Hasan Sarkar, Abu Sayeed Mohammad Mahmud, Mohammad Samir Uzzaman, Eshrar Osman, Md. Ahasan Habib, Shahina Akter, Taninina
Akhter Banu, Barna Goswami, Iffat Jahan, Md. Saddam Hossain, Mohammad Mohi Uddin, Md. Kamrul Islam, Mohammad Mizanur Rahman, Susane Giti.
Md. Salim Khan

1587438, EPL_SL_1587439, EPI_SL_1587440, EPI_ISL_1587441, EPIISL_1587442, EPI_SL_1587443, EPL_SL_1587444, EPI_ISL_1587445,

Hassan Afrad, Sadia Rahman, Fidausi Qadri, Tahmina Shirin

Abu Sayeed Mohammad Mahmud, Md. Murshed Hasan Sarkar, Mohammad Samir Uzzaman, Eshrar Osman, Md. Ahasan Habib, Shahina Akter, Tanjina
Akhter Banu, Barna Goswami, Iffat Jahan, Md. Saddam Hossain, Mohammad Mohi Uddin, Md. Kamrul Islam, Mohammad Mizanur Rahman, Susane Gitt, Abu Sayeed Mohammad Mahmud, Md. Murshed Hasan Sarkar, Mohammad Samir Uzzaman, Eshrar Osman, Md. Ahasan Habib, Shahina Akter, Taniina
Akhter Banu, Barna Goswami, Iffat Jahan, Md. Saddam Hossain, Mohammad Mohi Uddin, Md. Kamrul Islam, Mohammad Mizanur Rahman, Susane Giti.
Md. Salim Khan

Hassan Afrad, Sadia Rahman, Fidausi Qadri, Tahmina Shirin

Mohammad Mizanur Rahman, Md. Murshed Hasan Sarkar, Mohammad Samir Uzzaman, Eshrar Osman, Md. Ahasan Habib, Shahina Akter, Tanjina
Akhter Banu, Abu Sayeed Mohammad Mahmud, Barna Goswami, Iffat Jahan, Md. Saddam Hossain, Mohammad Mohi Uddin, Md. Kamrul Islam, Susane Susane Giti, Md. Murshed Hasan Sarkar, Mohammad Samir Uzzaman, Eshrar Osman, Md. Ahasan Habib, Shahina Akter, Tanjina Akhter Banu, Abu
Sayeed Mohammad Mahmud, Barna Goswami, Iffat Jahan, Md. Saddam Hossain, Mohammad Mohi Uddin, Md. Kamrul Islam, Mohammad Mizanur

Shahina Akter, Md. Murshed Hasan Sarkar, Mohammad Samir Uzzaman, Eshrar Osman, Md. Anasan Habib, Taninga Akhter Banu, Abu Sayeed
Mohammad Mahmud, Barna Goswami, Iffat Jahan, Md. Saddam Hossain, Mohammad Mohi Udddin, Md. Kamrul Islam, Mohammad Mizanur Rahman, Susane Giti, Md. Salim Khan

Tanjina Akhter Banu, Md. Murshed Hasan Sarkar, Mohammad Samir Uzzaman, Eshrar Osman, Md. Ahasan Habib, Shahina Akter, Tanjina Akhter Banu,
Abu Sayeed Mohammad Mahmud, Barna Goswami, Iffat Jahan, Md. Saddam Hossain, Mohammad Mohi Uddin, Md. Kamrul Islam, Mohammad Mizanur Rahman, Susane Gitit, Md. Salim Khan

Md. Murshed Hasan Sarkar, Mohammad Samir Uzzaman, Eshrar Osman, Md. Ahasan Habib, Shahina Akter, Tanjina Akkter Banu, Abu Sayeed
Mohammad Mahmud, Barna Goswami, Iffat Jahan, Md. Saddam Hossain. Mohammad Mohi Uddin, Md. Kamrul Islam, Mohammad Mizanur Rahman, Susane Giti, Md. Salim Khan Barna Goswami, Md. Murshed Hasan Sarkar, Mohammad Samir Uzzaman, Eshrar Osman, Md. Ahasan Habib, Shahina Akter, Tanjina Akhter Banu, Abu
Sayeed Mohammad Mahmud, Iffat Jahan, Md. Saddam Hossain, Mohammad Mohi Uddin, Md. Kamrul Islam, Mohammad Mizanur Rahman, Susane Gitt. Iffat Jahan, Md. Murshed Hasan Sarkar, Mohammad Samir Uzzaman, Eshrar Osman, Md. Ahasan Habib, Shahina Akter, Taninina Akhter Banu, Abu
Sayeed Mohammad Mahmud, Barna Goswami, Md. Saddam Hossain, Mohammad Mohi Uddin, Md. Kamrul Islam, Mohammad Mizanur Rahman, Susane
Giti, Md. Salim Khan 
EPL_ISL_1595841, EPI_SL_1595842

EPI_ISL_1595843

EPI_ISL_1615663, EPI_ISL_1615664

EPI_ISL_1616000, EPI_ISL_1616606

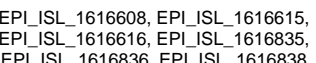
EPI ISL_1629810, EPI_ISL 1629811
Md. Saddam Hossain, Md. Murshed Hasan Sarkar, Mohammad Samir Uzzaman, Essrar Osman, Md. Ahasan Habib, Shahina Akter, Taninina Akhter Banu,
Abu Sayeed Mohammad Mahmud, Barna Goswami, Iffat Jahan, Mohammad Mohi Uddin, Md. Kamrul Islam, Mohammad Mizanur Rahman, Susane Giti,
Md. Salim Khan Mohammad Mohi Uddin, Md. Murshed Hasan Sarkar, Mohammad Samir Uzzaman, Eshrar Osmann, Md. Ahasan Habib, Shahina Akter, Tanjina Akhter
Banu, Abu Sayeed Mohammad Mahmud, Barna Goswami, Iffat Jahanan, Md. Saddam Hossain, Md. Kamul Islam, Mohammad Mizanur Rahman, Susane Md. Murshed Hasan Sarkar, Mohammad Samir Uzzaman, Eshrar Osman, Md. Ahasan Habib, Shahina Akter, Tanjina Akhter Banu, Abu Sayeed
Mohammad Mahmud, Barna Goswami, Iffat Jahan, Md. Saddam Hossain, Mohammad Mohi Uddin, Md. Kamrul Islam, Mohammad Mizanur Rahman, Susane Giti, Md. Salim Khan

Mohammad Mizanur Rahman, Md. Murshed Hasan Sarkar, Mohammad Samir Uzzaman, Eshrar Osman, Md. Ahasan Habib, Shahina Akter, Tanjina Wami, Iffat Jahan, Md. Saddam Hossain, Mohammad Mohi Uddin, Md. Kamrul Islam, Susan Susane Giti, Md. Murshed Hasan Sarkar, Mohammad Samir Uzzaman, Eshrar Osman, Md. Ahasan Habib, Shahina Akter, Taninina Akhter Banu, Abu
Sayeed Mohammad Mahmud, Barna Goswami, Iffat Jahan, Md. Saddam Hossain, Mohammad Mohi Uddin, Md. Kamrul Islam, Mohammad Mizanu Rahman, Md. Salim Khan

Md. Murshed Hasan Sarkar, Mohammad Samir Uzzaman, Eshrar Osman, Md. Ahasan Habib, Shahina Akter, Tanina Akhter Banu, Abu Sayeed Mohammad Mahmud, Barna Goswami, Iffat Jahan, Md. Saddam Hossain, Mohammad Mohi Uddin, Md. Kamrul Islam, Mohammad Mizanur Rahman, Md. Murshed Hasan Sarkar, Mohammad Samir Uzzaman, Eshrar Osman, Md. Ahasan Habib, Shahina Akter, Taninga Akhter Banu, Abu Sayeed
Mohammad Mahmud, Barna Goswami, Iffat Jahan, Md. Saddam Hossain, Mohammad Mohi Uddin, Md. Kamrul Islam, Mohammad Mizanur Rahman,
Susane Giti, Md. Salim Khan

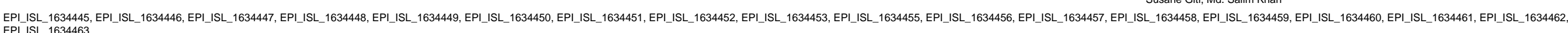

see above
EPI_ISL_1636523, EPI_ISL_1653815

EPI_ISL_1653893, EPI_ISL_1653894,
EPIISL-1659302, EPIISL-1653921,

(1)

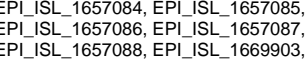

EPI_ISL_1657088, EPI_ISL-1669903,
EPIIIL_166990, EPIISL-1669905,
EPI_ISL_1669906, EPI_ISL_1669907

EPI_ISL_1673289

EPI_ISL_1673292

EPI_ISL_1673302

EPI_ISL_1673334, EPI_ISL_1673339,
EPI_ISL_1673343, EPI_ISL_1673415

EPI_ISL_1673673

EPI_ISL_1673680

EPIIISL11714800, EPIIISL_1714801,
EPI_ISL_1714805, EPI_ISL_1714806

EPI_ISL_1715160, EPI_ISL_1715161, EP _

see above

Child Health Research Foundation

DNA Solution Ltd.

Child Health Research Foundation

Genomic Research Lab, BCSIR

DNA Solution Ltd.

Genomic Research Lab, BCSIR

DNA Solution Ltd.

Genomic Research Lab, BCSIR

Institute for Developing Science and Health Inititatives (ideSHi) Institute for Developing Science and Health Initiatives (ideSHI)
DNA Solution Ltd.

DNA Solution Ltd.

DNA Solution Ltd.

DNA Solution Ltd.

Genomic Research Lab, BCSIP

Genomic Research Lab, BCSIR

GRMDCR \& JU
Genomic Research Lab, BCSIR

Genomic Research Lab, BCSIR

Genomic Research Lab, BCSIR

Genomic Research Lab, BCSIR

Genomic Research Lab, BCSIR

Genomic Research Lab, BCSIR

Genomic Research Lab, BCSIR
CHRF Bangladesh Genomics Tean

Md. Murshed Hasan Sarkar, Mohammad Samir Uzzaman, Eshrar Osman, Md. Ahasan Habib, Shahina Akter, Tanina Akhter Banu, Abu Sayeed
Mohammas Nadim Hasan, Md. Mizanur Rahman, Md. Abdul Khaleque, Sharif Akhteruzzamani, Md. Salim Khan

Mohammad Fazle Alam Rabbi, Md. Murshed Hasan Sarkar, Mohammad Samir Uzzaman, Eshrar Osman, Md. Ahasan Habib, Shahina Akter, Taniina
khhter Banu, Abu Sayeed Mohammad Mahmud, Barna Goswami, Iffat Jahan, Md. Saddam Hossain, Mohammad Mohi Uddin, Md Firoz Kabir, Kazi Nadiin 政

Abu Sayeed Mohammad Mahmud, Md. Murshed Hasan Sarkar, Mohammad Samir Uzzaman, Eshrar Osman, Md. Ahasan Habib, Shahina Akter, Tanjina
Akhter Banu, Barna Goswami, Iffat Jahan, Md. Saddam Hossain, Mohammad Mohi Uddiin, Mohammad Fazle Alam Rabbi, Md Firoz Kabir, Kazi Nadim Hasan, Md. Mizanur Rahman, Md. Abdul Khaleque, Sharif Akhteruzzamani, Md. Salim Khan Hassan Afrad, Sadia Rahman, Fidausi Qadri, Tahmina Shirin Barna Goswami, Md. Murshed Hasan Sarkar, Mohammad Samir Uzzaman, Eshrar Osman, Md. Ahasan Habib, Shahina Akter, Taninina Akhter Banu, Abu
Sayeed Mohammad Mahmudlffat Jahan, Md. Saddam Hossain, Mohammad Mohi Uddin, Mohammad Fazle Alam Rabbi, Md Firoz Kabir, Kazi Nadim
Hasan, Md. Mizanur Rahman, Md. Abdul Khaleque, Sharifi Akhteruzzamani, Md. Salim Khan Iffat Jahan, Md. Murshed Hasan Sarkar, Mohammad Samir Uzzaman, Estrar Osman, Md. Ahasan Habib, Shahina Akter, Taninina Akhter Banu, Abu
Sayeed Mohammad Mahmud, Barna Goswami, Md. Saddam Hossain, Mohammad Mohi Uddin, Mohammad Fazle Alam, Rabbi, Md Firoz Kabir, Kazi
Nadim Hasan, Md. Mizanur Rahman, Md. Abdul Khaleque, Sharifi Akhteruzzamani, Md. Salim Khan Md. Saddam Hossain, Md. Murshed Hasan Sarkar, Mohammad Samir Uzzaman, Eshrar Osman, Md. Ahasan Habib, Shahina Akter, Tanjina Akhter Banu,
Abu Sayeed Mohammad Mahmudd, Barna Goswami, Iffat Jahan, Mohammad Mhhi dddin, Mohammad Fazle Alam Rabbi, Md Firoz Kabir, Kazi Nadim
Hasan, Md. Mizanur Rahman, Md. Abdul Khaleque, Sharif Akhteruzzamani, Md. Salim Khan

Md. Murshed Hasan Sarkar, Mohammad Samir Uzzaman, Eshrar Osman, Md. Ahasan Habib, Shahina Akter, Tanina Akhter Banu, Abu Sayeed
Mohammad Mahmud, Barna Goswami, Iffat Jahan, Md. Saddam Hossain, Mohammad Mohi Uddin, Mohammad Fazle Alam Rabbi, Md Firoz Kabir, Kaz Nadim Hasan, Md. Mizanur Rahman, Md. Abdul Khaleque, Sharif Akhteruzzamani, Md. Salim Khan Nadiim Hasan, Md. Mizanur Rahman, Md. Abdul Khaleque, Sharif Akhteruzzamani, Md. Salim Khan
Taninina Akhter Banu, Md. Murshed Hasan Sarkar, Mohammad Samir Uzzaman, Eshrar Osman, Mdd Ahasan Habib, Shahina Akter, Abu Sayeed
Mohammad Mahmud, Barna Goswami, Iffat Jahan, Md. Saddam Hossain, Mohammad Mohi Uddin, Mohammad Fazle Alam Rabbi, Md Firoz Kabir, Kazz
Nadim Hasan, Md. Mizanur Rahman, Md. Abdu Khaleque, Sharif Akhteruzzamani, Md. Salim Khan

Tanjina Akhter Banu, Md. Murshed Hasan Sarkar, Mohammad Samir Uzzaman, Eshrar Osman, Md. Ahasan Habib, Shahina Akter, Abu Sayeed
Mohammad Mahmud, Barna Goswami, Iffat Jahan, Md. Saddam Hossain, Mohammad Mohi Uddin, Md. Salim Khan

Md. Murshed Hasan Sarkar, Mohammad Samir Uzzaman, Eshrar Osman, Md. Ahasan Habib, Shahina Akter, Tanjina Akhter Banu, Abu Sayeed

Mohammad Mahmud, Barna Goswami, Iffit Jahan, Md. Saddam Hossain, Mohammad Mohi Uddin, Nihad Adnan, Mohd Raeed Jamiruddin, M Ahsanul
Haq, Mohib Ullah Khondoker, Maha Jamiruddin, Md. Rubel Hossain, Nowshin Jahan, Tamanna Ali, Shahad Saif Khandker, M Firoz Ahmed, Md. Salim

PI_ISL_1715169, EPI_ISL_1715170, EPI_ISL_1717025

Mohammad Enayet Hossain, Mojnu Miah, Rashedul Hasan, Md. Mahfuzur Rahman, Mohammed Ziaur Rahman, Mustafizur Rahman

Shahina Akter, Md. Murshed Hasan Sarkar, Mohammad Samir Uzzaman, Eshrar Osman, Md. Ahasan Habib, Tanina Akhter Banu, Abu Sayeed Mohammad Mahmud, Barna Goswami, Iffat Jahan, Md. Saddam Hossain, Mohammad Mohi Uddin, Nihad Adnan, Mohd Raeed Jamiruddin, M Ahsanul
Haq, Mohib Ullah Khondoker, Maha Jamiruddin, Mdd. Rubel Hossain, Nowshin Iahan, Tamanna Ali, Shahad Saif Khandker, M Firoz Ahmed, Md. Salim

Tanjina Akhter Banu, Md. Murshed Hasan Sarkar, Mohammad Samir Uzzaman, Eshrar Osman, Md. Ahasan Habib, Shahina Akter, Abu Sayeed

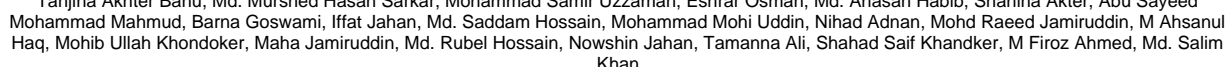
Tanjina Akhter Banu, Md. Murshed Hasan Sarkar, Mohammad Samir Uzzaman, Eshrar Osman, Md. Ahasan Habib, Shahina Akter, Abu Sayeed Mohammad Mahmud, Barna Goswami, Iffat Jahan, Md. Saddam Hossain, Mohammad Mohi Uddin, Md. Kamrul Islam, Nihad Adanan, Mohd Raeed
Jamiruddin, M Ahsanul Haq, Mohib Ullah Khondoker, Maha Jamiruddin, Md. Rubel Hossain, Nowshin Jahan, Tamanna Ali, Shahad Saif Khandker, M Firoz Abu Sayeed Mohammad Mahmud, Md. Murshed Hasan Sarkar, Mohammad Samir Uzzaman, Eshrar Osman, Md. Ahasan Habib, Shahina Akter, Tanjina 
EPI_ISL_1742837, EPI_ISL1742838,
EPI_ISL_1743263, EPI_ISL_1743591

EPI_ISL_1750956, EPI_ISL_1750957, LSL-1750960, EPIIISL-1750961,
EPI_ISL_1750962

EPI_ISL_1752689, EPI_ISL_1752690, EPI_SL see above

EPI_ISL_1760648, EPI_ISL 1760649 ,
EPI_ISL_1760650 EP__ISL_1790209

EPI_ISL_1790213

EPI_ISL_1793784, EPI_ISL_1793785,

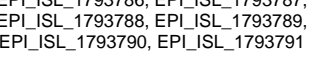
EPI_ISL_1805696

EPI_ISL_1805701

EPI_ISL_1825676, EPI_ISL_1825678,

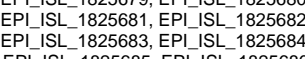
EPIISL_1828779, EPI_ISL-1828780, EPI_ISL_1915108, EPI_ISL_1915109, EPI_ see above

EPIIISL_1915436, EPI_ISL_1915437,
EPI_ISL_1915438, EPI_ISL_1915439 EPI_ISL_1920962

EPI_ISL_1938475, EPI_ISL 1938476,
EPI_ISL_1938477 EPI_ISL_1942249

EPI_ISL_2002482

EPL_ISL_2003459
GRMDCR \& JU

Genomic Research Lab, BCSIR
Akhter Banu, Barna Goswami, Iffat Jahan, Md. Saddam Hossain, Mohammad Mohi Uddin, Md. Kamrul Islam, Nihad Adnan, Mohd Raeed Jamiruddin, M
Ahsanul Haq, Mohib Ullah Khondoker, Maha Jamiruddin, Md. Rubel Hossain, Nowshin Jahan, Tamanna Ali, Shahad Saif Khandker, M Firoz Ahmed, Md. Hossain, Nows
Salim Khan

Barna Goswami, Md. Murshed Hasan Sarkar, Mohammad Samir Uzzaman, Eshrar Osman, Md. Ahasan Habib, Shahina Akter, Tanjina Akhter Banu, Abu

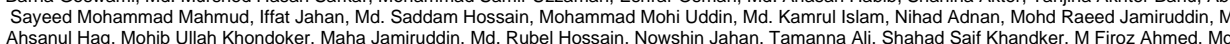
Salim Khan

Iffat Jahan, Md. Murshed Hasan Sarkar, Mohammad Samir Uzzaman, Eshrar Osman, Md. Ahasan Habib, Shahina Akter, Tanjina Akhter Banu, Abu Sayeed Mohammad Mahmud, Barna Goswami, Md. Saddam Hossain, Mohammad Mohi Uddin, Md. Kamrul Islam, Nihad Adnan, Mohd Raeed Jamirudd

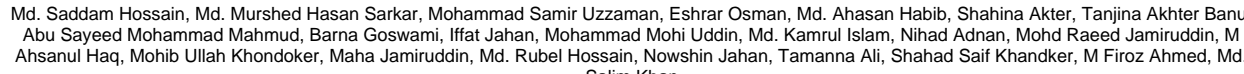
im Khan

Md. Murshed Hasan Sarkar, Mohammad Samir Uzzaman, Eshrar Osman. Md. Ahasan Habib, Shahina Akter, Taninina Akhter Banu, Abu Sayeed Mohammad Mahmud, Barna Goswami, Iffat Jahanan, Md. Saddam Hossain, Mohammad Mohi Uddin, Md. Karrul Islam, Nihad Adnan, Mohd Raeed
aniruddin, M Ahsanul Haq, Mohib Ullah Khondoker, Maha Jamiruddin, Mdd. Rubel Hossain, Nowshin Jahan, Tamanna Ali, Shahad Saif Khandker, M Firoz

Hassan Afrad, Sadia Rahman, Fidausi Qadri, Tahmina Shirin

Institute of Epidemiology, Disease Control and Research Institute for Developing Science and Health Initiatives (ideSHi)
(IEDCR) 752691, EPI_ISL_1752692, EPI_ISL_1752693, EPI_ISL_1752694, EP__ISL_1752702, EPI_ISL_1752703, EPI_ISL_1752704, EPI_ISL_1752705, EPI_ISL_1752706, EPI_ISL_1752707 Child Health Research Foundation Child Health Research Foundation Institute of Epidemiology, Disease Control and Research Institute for Developing Science and Health Initiatives (ideSHi)
(IEDCR) Department of Genetic Engineering and Biotechnology, Genomic Research Lab, BCSIR
Shahialal University of Science and Technology

(a)

\section{Department of Genetic Engineering and Biotechnology,
Shahijalal University of Science and Technology}

Genomic Research Lab, BCSIR

\section{Virology Laboratory, International Centre for Diarrhoeal
Disease Research, Bangladesh (ICDDR,B)}

Department of Genetic Engineering and Biotechnology,
Shahjalal University of Science and Technology

\section{Virology Laboratory, International Centre for Diarrhoea
Disease Research, Bangladesh (ICDDR,B)}

Genomic Research Lab, BCSIR

Genomic Research Lab, BCSIR
CHRF Bangladesh Genomics Team Hassan Afrad, Sadia Rahman, Fidausi Qadri, Tahmina Shirin

Md. Murshed Hasan Sarkar, Abu Sayeed Mohammad Mahmud, Mohammad Samir Uzzaman, Eshrar Osman, Md. Ahasan Habib, Shahina Akter, Tanjina Akhter Banu, Barna Goswami, Iffat Jahan, Md. Saddam Hossain, Mohammad Mohi Uddin, Md. Kamrul Islam, Md. Shamsul Haque Prodhan, Md.
Hammadul Hoque, G. M. Nurnabi Azad Jewel, Md. Nazmul Hasan, Md. Fahmid Hossain Bhuiyan, Md. Asraful Jahan, Ajt Ghosh, Md. Akkas Ali, Md. Salim
Khan Tanina Akhter Banu, Md. Murshed Hasan Sarkar, Abu Sayeed Mohammad Mahmud, Mohammad Samir Uzzaman, Eshrar Osman, Md. Ahasan Habib,
Shahina Akter, Barna Goswami, Iffat Jahan, Md. Saddam Hossain, Mohammad Mohi Uddin, Md. Kamrul Islam, Md. Shamsul Haque Prodhan, Md. Shahina Akter, Barna Goswami, Iffat Jahan, Md. Saddam Hossain, Mohammad Mohi Uddin, Md. Kamrul Islam, Md. Shamsul Haque Prodhan, Md.
Hammadul Hoque, G. M. Nurnabi Azad Jewel, Md. Nazmul Hasan, Md. Fahmid Hossain Buuiyan, Md. Asraful Jahan, Aijt Ghosh, Md. Akkas Ali, Md. Salim
Khan

Mohammad Enayet Hossain, Mojnu Miah, Rashedul Hasan, Md. Mahtuzur Rahman, Mohammed Ziaur Rahman, Mustafizur Rahman Md. Murshed Hasan Sarkar, Abu Sayeed Mohammad Mahmud, Mohammad Samir Uzzaman, Eshrar Osman, Md. Ahasan Habib, Shahina Akter, Tanjina
Akhter Banu, Barna Goswami, Iffat Jahan, Md. Saddam Hossain, Mohammad Mohi Iddin, Md. Kamrul slam, Md. Shambul Haque Prodhan, Md.
Hammadul Hoque, G. M. Nurnabi Azad Jewel, Md. Nazmul Hasan, Md. Fahmid Hossain Bhuiyan, Md. Astraful Jahan, Ajit Ghosh, Md. Akkas Ali, Md. Salim Kammadul Hoque, G. M. Nurnabi Azad Jewel, Md. Nazmul Hasan, Md. Fahmid Khan

Md. Salim Khan, Md. Murshed Hasan Sarkar, Abu Sayeed Mohammad Mahmud, Mohammad Samir Uzzaman, Eshrar Osman, Md. Ahasan Habib,

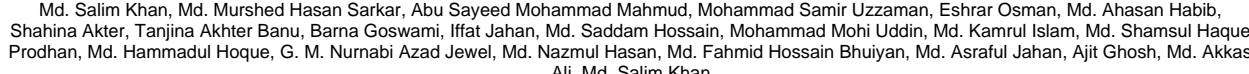
Goutam Buddha Das, Tridip Das, Tanvir Ahmad Nizami, Eaftekhar Ahmed Rana, Md. Sirazul Islam, Prone
Hasan Sarkar, Md. Salim Khan, Paritosh Kumar Biswas

COVID-19 Detection Lab, Chattogram Veterinary and Animal Genomic Research Lab, Bangladesh Cou
Sciences University
Industrial Research

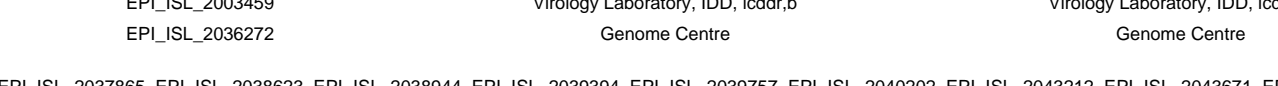

Virology Laboratory, International Centre for Diarrhoeal
Disease Research, Bangladesh (ICDDR,B) 19110, EPI_ISL_1915111, EPI_ISL_1915112, EPI_ISL_1915113, EPI_ISL_1915114, EPI_ISL_1915115, EPI_ISL_1915116, Institute for Developing Science and Health Initiatives (ideSHi) Institute for Developing Science and Health Initiatives (ideSHi) Institute of Epidemiology, Disease Control and Research Institute for Developing Science and Health Initiatives (ideSHi) Institute for Developing Science and Health Initiatives (ideSHi) Institute for Developing Science and Health Initiatives (ideSHi) Institute of Epidemiology, Disease Control and Research Institute for Developing Science and Health Initiatives (ideSHi)
(IEDCR)

$\begin{array}{cc}\text { Genome Centre } & \text { Genome Centre } \\ \text { Gomic Research Lab, BCSIR } & \text { Genomic Research Lab, BCSIR } \\ \text { Genome Centre } & \text { Virology Laboratory, IDD, icddr,b } \\ \text { Genome Centre }\end{array}$
$\begin{array}{ll}\text { see above } & \text { Biological Hazard and Health Research Laboratory, Centre for } \\ \text { Advanced Research in Science (CARS), University of Dhaka }\end{array}$

EPI_ISL_2105565 Genomic Research Lab, BCSIR

Institute of Epidemiology, Disease Control and Research ideSHi-IEDCR-icddr,b
(IEDCR)
Mohammad Enayet Hossain, Mojnu Miah, Rashedul Hasan, Md. Mahfuzur Rahman, Mohammed Ziaur Rahman, Mustafizur Rahman PI_ISL_1915117, EPI_ISL_1915118, EPI_ISL_1915119, EPI_ISL_1915120

Hassan Atrad, Sadi Rahman, Fidausi Oadri Tahmina Shin Hassan Afrad, Sadia Rahman, Fidausi Qadri, Tahmina Shirin

Hassan Afrad, Sadia Rahman, Fidausi Qadri, Tahmina Shirin Hassan Afrad, Sadia Rahman, Fidausi Oadri, Tahmina Shiri Md. Shazid Hasan, Shovon Lal Sarkar, Ali Ansan Setu, Tanay Chakrabarty, A. S. M. Rubayet UI Alam, M. Shaminur Rahman, Ovinu Kibria Islam, M. Tanvir
Islam, Hassan M. Al-Emran, labal Kabir Jahid, M. Anwar Hossain

Md. Murshed Hasan Sarkar, Abu Sayeed Mohammad Mahmud, Mohammad Samir Uzzaman, Eshrar Osman, Md. Ahasan Habib, Shahina Akter, Tanin

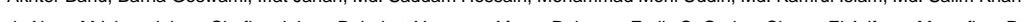
Shovon Lal Sarkar, Md. Shazid Hasan, Ali Ahsan Setu, Prosanto Kumar Das, A. S. M. Rubayet UI Alam, Ovinu Kibria Islam, Md. Tanvir Islam, Hassan Md.

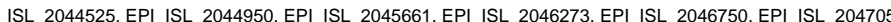

Md Mizanur Rahaman, Md Abdul Malek, Md. Shaminur Rahman, Latiful Bari, Md. Murshed Hasan Sarkar, Abu Sayeed Mohammad Mahmud, Mohammad
Samir Uzzaman, Eshrar Osman, Md. Ahasan Habib, Shahina Akter, Tanjina Akhter Banu, Barna Goswami, Iffat Jahan, Md. Saddam Hossain, Mohammad Shahina Akter, Taninina Akhter Banu, Barna Gos
Mohi Uddin, Md. Kamrul Islam, Md. Salim Khan 


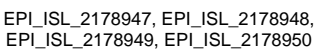
EPIISL_2178951

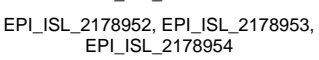

EPLISL_2178955, EPIIISL2178956,
EPI_ISL_2178957

EPI_ISL_2178958

EPI_ISL_2178959

see above

see above
EPI_ISL_2179666, EPI_ISL_2179667

EPI_ISL_2180454, EPI_ISL2180455,
EPI_ISL_2180456, EPI_ISL_2180457

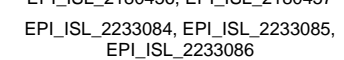

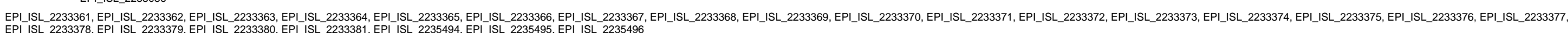
see above Virology Laboratory, International Centre for Diarrhoe
Disease Research, Bangladesh (ICDDR,B)

Virology Laboratory, International Centre for Diarrhoeal
Disease Research, Bangladesh (ICDDR,B)

EPP_ISL_2284885, EPI_ISL_2228888, EPI_ISL_2284887,

\begin{tabular}{|c|c|c|}
\hline see above & $\begin{array}{l}\text { Institute of Epidemiology, Disease Control and Research } \\
\text { (IEDCR) }\end{array}$ & IEDCR-ideSHi-icddr,b \\
\hline EPI_ISL_2348652 & Genome Center & Genome Center \\
\hline EPI_ISL_2348658 & Genome Center & Genome Center \\
\hline EPI_ISL_2348660 & Genome Center & Genome Center \\
\hline EPI_ISL_2348661 & Genome Center & Genome Center \\
\hline EPI_ISL_2348662 & Genome Center & Genome Center \\
\hline EPI_ISL_2348663 & Genome Center & Genome Center \\
\hline EPI_ISL_2348664 & Genome Center & Genome Center \\
\hline EPI_ISL_2349692 & Genome Center & Genome Center \\
\hline EPI_ISL_2349879 & Genome Center & Genome Center \\
\hline EPI_ISL_2349880 & Genome Center & Genome Center \\
\hline EPI_ISL_2350142 & Genome Center & Genome Center \\
\hline EPI_ISL_2350167 & Genome Center & Genome Center \\
\hline
\end{tabular}

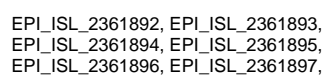

Virology Laboratory, International Centre for Diarrhoeal
Disease Research, Bangladesh (ICDDR,B)

Virology Laboratory, International Centre for Diarrhoeal
Disease Research, Bangladesh (ICDDR,B)
CHRF Bangladesh Genomics Team, Zaheed Husain, Shafiul Azam

CHRF Bangladesh Genomics Team

CHRF Bangladesh Genomics Team, Zaheed Husain, Shafiul Azam

CHRF Bangladesh Genomics Team

CHRF Bangladesh Genomics Team, Zaheed Husain, Shafiul Azam

CHRF Bangladesh Genomics Team
192_2178971, EPI_ISL_2178972, EPI_ISL_2178973, EPI_ISL_2178974, EPI_ISL_2178975

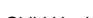

CHRF Bangladesh Genomics Team, Zaheed Husain, Shafiul Azam

Hassan Afrad, Sadia Rahman, Manjur Hossain Khan, Firdausi Qadri, Tahmina Shirin

Hassan Afrad, Sadia Rahman, Manjur Hossain Khan, Firdausi Qadri, Tahmina Shirin

Hassan Afrad, Sadia Rahman, Manjur Hossain Khan, Firdausi Qadri, Tahmina Shirin
Mohammad Enayet Hossain, Mojnu Miah, Rashedul Hasan, Md. Mahfuzur Rahman, Mohammed Ziaur Rahman, Mustafizur Rahman 2304468

Hassan Afrad, Sadia Rahman, Manjur Hossain Khan, Firdausi Qadri, Tahmina Shirin

Shovon Lal Sarkar, Md. Shazid Hasan, Ali Ansan Setu, Tanay Chakrabarty, Prosanto Kumar Das, A. S. M. Rubayet UI Alam, Ovinu Kibria Islam, Md. Tanvir
Islam, Hassan Md. Al-Emran, Iqbal Kabir Jahid, M. Anwar Hossain Md. Shazid Hasan, A. S. M. Rubayet UI Alam, Shovon Lal Sarkar, Ali Ahsan Setu, Tanay Chakrabarty,Prosanto Kumar Das, M. Shaminur Rahman, Ovinu A. S. M. Rubayet UI Alam, Md. Shazid Hasan, Shovon Lal Sarkar, Ali Ahsan Setu, Tanay Chakrabarty, Prosanto Kumar Das, M. Shaminur Rahman, Ovinu
Kibria Islam, M. Tanvir Islam, Hassan M. Al-Emran, Ilabal Kabir Jahid, M. Anwar Hossain M. Tanvir Islam, A. S. M. Rubayet UI Alam, Md. Shazid Hasan, Shovon Lal Sarkar, Ali Ahsan Setu, Tanay Chakrabarty,Prosanto Kumar Das, M. Shaminur

Ovinu Kibria Islam, M. Tanvir Islam, A. S. M. Rubayet UI Alam, Md. Shazid Hasan, Shovon Lal Sarkar, Ali Ahsan Setu, Tanay Chakrabarty, Prosanto Kumar

Iqbal Kabir Jahid, Md. Shazid Hasan, A. S. M. Rubayet Ul Alam, Shovon Lal Sarkar, Ali Ahsan Setu, Tanay Chakrabarty, Prosanto Kumar Das, M.
Shaminur Rahman, Ovinu Kibria Islam, M. Tanvir Islamm, Hassan M. Al-Emran, M. Anwar Hossain

Hassan Md. Al-Emran, Shovon Lal Sarkar, Md. Shazid Hasan, Ali Ansan Setu,Tanay Chakrabarty,Prosanto Kumar Das, A. S. M. Rubayet UI Alam, Ovinu
Kibria Islam, Md. Tanvir Islam, Iqbal Kabir Jahid, M. Anwar Hossain

Ovinu Kibria Islam, Hassan Md. Al-Emran, Shovon Lal Sarkar, Md. Shazid Hasan, Ali Ahsan Setu, Tanay Chakrabarty, Prosanto Kumar Das, A. S. M.
Rubayet UI Alam, Md. Tanvir Islam, I labal Kabir Jahid, M. Anwar Hossain

Md. Tanvir Islam, Hassan Md. Al-Emran, Shovon Lal Sarkar, Md. Shazid Hasan, Ali Ansan Setu, Tanay Chakrabarty, Prosanto Kumar Das, A. S. M.
Rubayet UI Alam, Ovinu Kibria Islam, labal Kabir Jahid, M. Anwar Hossain Hassan Md. Al-Emran, Shovon Lal Sarkar, Md. Shazid Hasan, Ali Ansan Setu, Tanay Chakrabarty, Prosanto Kumar Das, A. S. M. Rubayet UI Alam, Ovinu A. S. M. Rubayet UI Alam, Md. Shazid Hasan, Shovon Lal Sarkar, Ali Ahsan Setu, Tanay Chakrabarty, Prosanto Kumar Das, M. Shaminur Rahman, Ovinu Md. Shazid Hasan, Shovon Lal Sarkar, Ali Ahsan Setu, A. S. M. Rubayet Ul Alam, Tanay Chakrabarty, Prosanto Kumar Das, M. Shaminur Rahman, Ovinu
Kibria Islam, M. Tanvir Islam, Hassan M. Al-Emran, Iqbal Kabir Jahid, M. Anwar Hossain Mohammad Enayet Hossain, Mojnu Miah, Rashedul Hasan, Md. Mahfuzur Rahman, Mohammed Ziaur Rahman, Mustafizur Rahman

EPI_ISL_2361896, EPI_ISL_23689

EPI_ISL_2399440, EPI_ISL_2399441, EPI_ISL_2399442, EPI_ISL_2399443, EPI_ISL_2399444, EPI_ISL_239944 see above Institute of Epidemiology, Disease Control and Research
(IEDCR) see above Institute of Epidemiology, Disease Control and Research
(IEDCR) see above Institute of Epidemiology, Disease Control and Research
(IEDCR)

, EPI_ISL_2399451, EPL_ISL_2399452, EPI_ISL_2399453, EPI_ISL_2399454, EPI_ISL_2399455, EPI_ISL_2399456 Hassan Afrad, Sadia Rahman, Manjur Hossain Khan, Firdausi Qadri, Tahmina Shirin 